МАШИН03НАВСТВO

УДК 621.865.8(031)

DOI https://doi.org/10.32838/2663-5941/2019.3-1/01

\title{
Полищук М.Н.
}

Национальный технический университет Украины

«Киевский политехнический институт имени Игоря Сикорского»

\section{ТЕОРЕТИЧЕСКОЕ ОБОСНОВАНИЕ ФУНКЦИОНИРОВАНИЯ АНТИГРАВИТАЦИОННОГО МОБИЛЬНОГО РОБОТА}

В работе изложено описание принципиально новой конструкции мобильного робота с генератором пневматической реактивной тяги, установленном на Кардановом подвесе. Приведены синтез конструкиий исполнительных механизмов робота и математические модели их функционирования. Техническое решение направлено на компенсачию или полное преодоление гравитационной нагрузки мобильного робота произвольной ориентачии при выполнении транспортных и технологических операщий. В совокупности изложенные решения способствуют повышению надёжности удержания мобильных роботов на поверхности произвольной ориентации в пространстве при их эксплуатации в различных областях промышленности.

Ключевые слова: мобильные роботы, шагающие механизмы, роботы вертикального перемещения, альпинистские роботы.

Постановка проблемы. Мобильные роботы произвольной ориентации в пространстве или роботы вертикального перемещения, известные в зарубежных публикациях как Climber Robot (альпинистский робот), предназначены для выполнения технологических операций в экстремальных условиях производства, в том числе и техногенных катастроф, опасных и даже неприемлемых для пребывания человека. Главным требованием к данному типу роботов является удержание их на поверхности перемещения. В экспериментальных образцах таких роботов, отмеченных ниже, данная задача решается созданием разнообразных подсистем сцепления робота с поверхностью перемещения. Здесь же предложен иной подход, а именно оснащение робота источником реактивной тяги нехимического происхождения, точнее пневматическим генератором аэродинамической подъемной силы, частично компенсирующей или полностью преодолевающей гравитационную нагрузку, не допуская при этом превращения мобильного робота в летательный аппарат, дабы обеспечить выполнение контактных силовых технологических операций при обслуживании различных промышленных объектов.

Анализ последних исследований и публикаций. В зависимости от топологии и физикомеханических свойств поверхности перемещения мобильных роботов применяются различные средства удержания робота под действием гравитационной нагрузки, а именно: вакуумные, механические, электромагнитные и основанные на явлении сухой или электрической адгезии $[1$, c. 37]. Однако, как и всем рациональным техническим решениям, каждому из них объективно свойственны преимущества и недостатки. Так, например, преимуществом вакуумных захватов мобильных роботов [2, с. $146 ; 3 ; 4$, с. 347-349; 5, с. 4-8] является их инвариантность к поверхности перемещения. Однако подток воздуха в зону вакуума при существенных перепадах топологии поверхности перемещения снижает надежность удержания робота, поэтому здесь уместна модернизация вакуумных захватов, предложенная в работе [6, с. 60]. Механические подсистемы сцепления [7, с. 11-13; 8, с. 42-44] отличаются высокой надежностью, но их приводы требуют применения редукции, что снижает удельную мощность робота, для компенсации которой целесообразно использование комбинированных приводов [9, с. 288-292] или систем накопления и преобразования энергии движения [10, с. 116-118]. Высокое быстродействие и простота конструкции свойственны электромагнитным устройствам сцепления $[11$, с. $5 ; 12$, с. 3], но преодоление силы гравитации за счет прилипания магнитов ограничивает применение данного средства только ферромагнитными поверхностями перемещения. 
Развитием подсистем сцепления робота с поверхностью перемещения являются технические решения [13, с. 6-8], в которых используется технология сцепления посредством адгезии. Однако современные реализации указанного сцепления с поверхностью перемещения характеризуются крайне низкой скоростью перемещения робота вследствие медлительности эффекта адгезии, что пока препятствует их промышленному использованию. Таким образом, проблема преодоления гравитационной нагрузки или, по крайней мере, частичной ее компенсации для мобильных роботов, способных выполнять технологические операции в пространстве произвольной ориентации, остается актуальной.

Постановка задачи. Для повышения надежности удержания робота на поверхности произвольной ориентации наряду с использованием известных подсистем сцепления робота с указанной поверхностью необходимо оснастить робот источником противодействия гравитационной нагрузке, действие которого не только противоположно по направлению, но и постоянно совпадает с линией силы гравитации независимо от положения робота в пространстве. Кроме того, необходимо, чтобы создаваемая реактивная тяга, т.е. аэродинамическая подъемная сила, не превышала усилие сцепления робота с поверхностью перемещения за счет регулирования указанной тяги в зависимости от угла наклона робота к горизонту технологического пространства.

Изложение основного материала исследования. Конструкция мобильного робота. В отличие от вышеизложенного, предложен иной способ

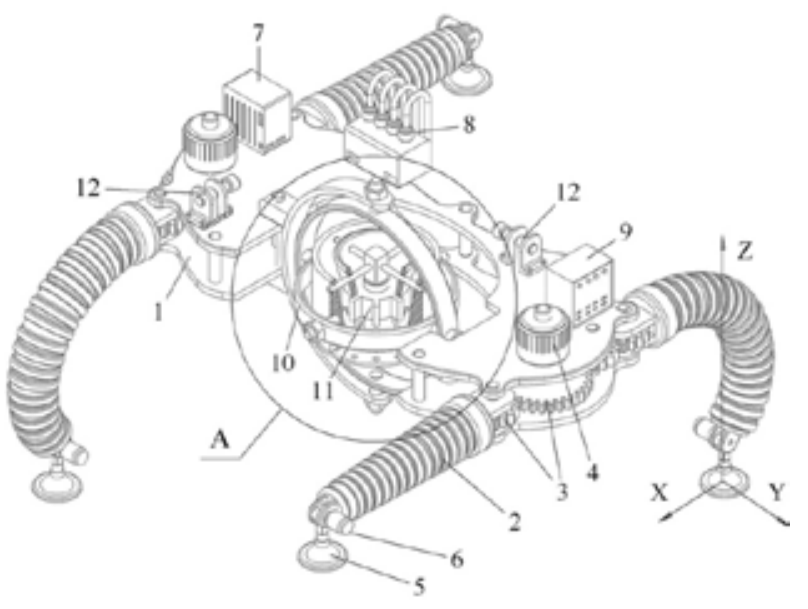

Рис. 1. Мобильный робот с генератором пневматической тяги противодействия гравитационной нагрузке робота произвольной ориентации, а именно использование аэродинамической подъемной силы, создаваемой генератором реактивной тяги нехимического происхождения. Именно в этом контексте (от лат. contextus - «соединение», «связь») применён термин антигравитация как способ только противодействия гравитационной нагрузке (но ни в коем случае не гашения её или экранирования), однако не превышающего усилия сцепления мобильного робота с поверхностью перемещения произвольной ориентации во избежание превращения робота в летательный аппарат.

Первоначально рассмотрим принципиально новую конструкцию мобильного робота с генератором пневматической реактивной тяги, установленном на Кардановом подвесе [14, с. 7-9]. На корпусе 1 (рис. 1) робота установлены шагающие механизмы - гибкие педипуляторы 2 , снабжённые зубчатой трансмиссией 3 и ее приводом 4. Наличие гибких педипуляторов позволяет осуществлять произвольную ориентацию робота в пространстве. На окончаниях указанных педипуляторов, принцип работы которых подробно изложен в работе $[15$, с. $5-8]$, установлены вакуумные (или любого иного типа) захваты 5 с приводами 6 для сцепления робота с поверхностью перемещения произвольной ориентации. Кроме того, робот оснащён блоком питания 7, комплектом пневматической и гидравлической аппаратуры 8 , а также котроллером управления 9. Однако принципиальным отличием мобильного робота является установка на Кардановом подвесе 10 с тремя степенями свободы генератора пневматической тяги 11.

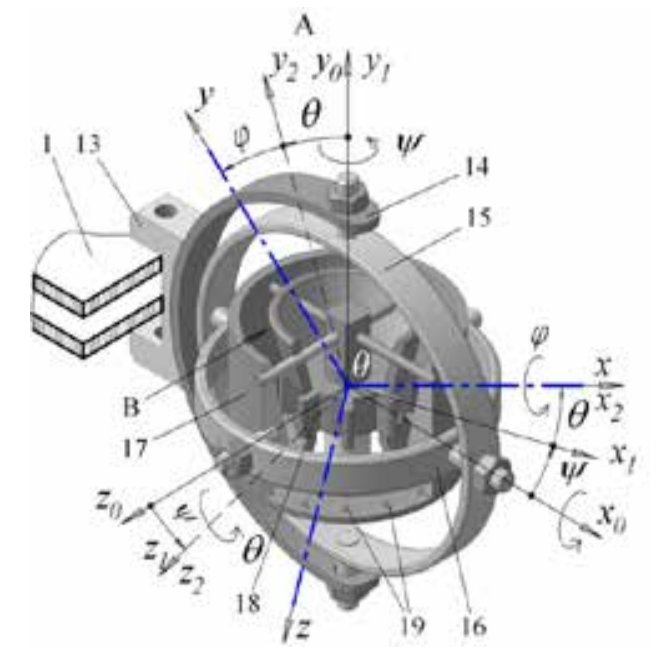

Рис. 2. Карданов подвес с тремя степенями свободы в виде углов поворота $\psi, \theta$ и $\varphi$ 
Устройства 12 для измерения угла наклона робота к горизонту выполнены в виде энкодеров - датчиков преобразования угла поворота в электрический сигнал, установленных на осях противовесов, всегда направленных под действием силы тяжести по направлению гравитационной силы.

Собственно, Карданов подвес 10, как показано выносным элементом «А» на рис. 2, установлен кронштейном 13 на корпусе 1 робота таким образом, что центры поворота его колец совпадают с центром масс робота. Подвес содержит неподвижное полукольцо 14 и два поворотных кольца внешнее 15 и внутреннее кольцо 16, установленные на соответствующих им осях. На внутреннем кольце 16 с возможностью поворота закреплён пневматический генератор тяги 17 (компрессор), лопастной ротор 18 которого, будучи снабжён сервоприводом и автономным блоком питания, всасывает воздух через коническую полость «В» и нагнетает сжатый воздух во множество сопел 19. Истекая из сопел, поток сжатого воздуха создаёт аэродинамическую подъёмную силу, направленную противоположно силе гравитации. Совокупность сопел создаёт суммарную подъёмную силу, поток воздуха в сопле является ламинарным при средней скорости воздуха в сопле не более $1 / 3$ скорости звука. Поскольку сам генератор тяги установлен с определённым смещением относительно центра Карданового подвеса, то возникающий при этом крутящий момент направляет ось генератора, а значит и создаваемый им поток сжатого воздуха, всегда по линии действия гравитационной силы тяжести и, естественно, противоположно последней. Силы сцепления мобильного робота с поверхностью перемещения зависят не только от физико-механических свойств поверхности перемещения, а прежде всего от нормальных реакций опор ног робота, которые, в свою очередь, определяются углом наклона робота к горизонту технологического пространства. Поэтому во избежание превышения реактивной тяги сил сцепления робота и недопустимости превращения его в летательный аппарат, а значит и утраты способности выполнять контактные силовые технологические операции, необходимо осуществлять регулирование производительности пневматического генератора тяги в зависимости от угла наклона робота к горизонту (рис. 3).

Такое регулирование осуществляется посредством связи указанного выше устройства измерения угла наклона робота с регулятором скорости сервопривода генератора тяги следующим образом. Сигналы энкодеров, пропорциональные углу

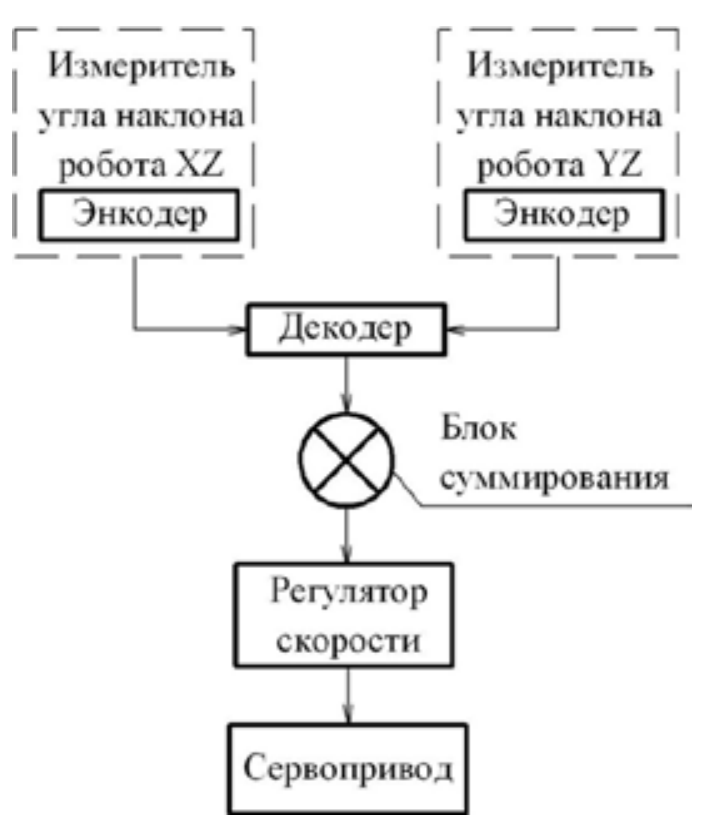

Рис. 3. Структурная схема блока управления

наклона робота, после дешифровки декодером и сравнения их в блоке суммирования поступают на регулятор скорости сервопривода генератора тяги, увеличивая или уменьшая производительность пневматического компрессора, и тем самым регулируют скорость истечения сжатого воздуха через множество сопел 19 (см. выше рис. 2). В итоге изменяется сила реактивной пневматической тяги в зависимости от углов наклона робота к горизонту, предупреждая тем самым возможность превышения сил сцепления робота с поверхностью перемещения. Таким образом повышается надёжность удержания робота на поверхности перемещения произвольной ориентации вне зависимости от типа захватов робота и физико-механических свойств топологии указанной поверхности. Для возможности проектной реализации подвеса генератора тяги выполним его кинематический и динамический анализ, а также расчёт подъёмной силы.

Кинематический анализ подвеса генератора тяги. Выберем в точке «O» (рис. 2) начало неподвижной системы координат $O x_{o} y_{o} z_{o}$ и начало подвижной системы координат $O x y z$, которая соединена с компрессором 17 и двигается вместе с ним. Компрессор имеет три степени свободы и для описания его движения удобно пользоваться углами Эйлера-Крылова $(\psi, \theta, \varphi)$, которые дают возможность, осуществив три поворота на заданные углы, получить необходимое положение соединённой с компрессором подвижной системы координат Oxyz. Первый поворот на угол $\psi$, кото- 
рый называется углом прецессии, осуществляем вокруг оси $O y_{o}$. При этом получаем систему координат $O x_{1} y_{1} z_{1}$, соединённую с компрессором и характеризующую его положение после первого поворота. Следующий поворот на угол $\theta$, который называется углом нутации, осуществляем вокруг оси $O z_{1}$ и получаем систему координат $O x_{2} y_{2} z_{2}$, также соединённую с компрессором. И наконец, третий поворот на угол $\varphi$, т.е. угол собственного поворота компрессора, осуществляем вокруг оси $O x_{2}$. В результате получаем окончательное положение компрессора, которое характеризуется системой координат Oxyz.

Записав для каждого из указанных поворотов матрицы $B_{1}, B_{2}, B_{3}$ направляющих косинусов осей относительно предыдущих систем координат и перемножив эти матрицы, получим матрицу $B$, столбцы которой являются направляющими косинусами осей $\mathrm{Ox}, \mathrm{Oy}, \mathrm{Oz}$ относительно неподвижной системы $O x_{o} y_{o} z_{o}$ :

$$
B=B_{1} B_{2} B_{3}=\left(\begin{array}{lll}
a_{11} & a_{12} & a_{13} \\
a_{21} & a_{22} & a_{23} \\
a_{31} & a_{32} & a_{33}
\end{array}\right),
$$

где обозначено: $a_{11}=\cos \theta \cos \psi ; a_{13}=\sin \psi \cos \varphi+$

$+\sin \varphi \cos \psi \sin \theta ; \quad a_{21}=\sin \theta ; \quad a_{22}=\cos \theta \cos \varphi ;$ $a_{23}=-\sin \varphi \cos \theta ; \quad a_{31}=-\sin \psi \cos \theta ; \quad a_{32}=\sin \varphi \cos \psi+$ $+\sin \psi \cos \varphi \sin \theta ; a_{33}=\cos \psi \cos \varphi-\sin \varphi \sin \psi \sin \theta$.

Каждому углу поворота $\psi, \theta, \psi$ соответствует вектор угловой скорости:

$\vec{\omega}_{1}=\vec{j}_{o} \frac{d \psi}{d t}=\dot{\psi} \vec{j}_{o} ; \quad \vec{\omega}_{2}=\vec{k}_{1} \frac{d \theta}{d t}=\dot{\theta} \vec{k}_{1} ; \quad \vec{\omega}_{3}=\vec{i} \frac{d \varphi}{d t}=\dot{\varphi} \vec{i}$,

где $\vec{j}_{o}, \vec{k}_{1}, \vec{i}$-единичные векторы, направленные соответственно вдоль координатных осей $O y_{o}, O z_{1}, O x$. Записав выражения суммарной угловой скорости и выразив единичные векторы $\vec{j}_{o}, \vec{k}_{1}$ через единичные векторы подвижной системы координат $\vec{i}, \vec{j}, \vec{k}$, находим проекции угловой скорости на оси подвижной системы координат:

$$
\begin{aligned}
& \omega_{x}=\dot{\varphi}+\dot{\psi} \sin \theta ; \quad \omega_{y}=\dot{\theta} \sin \varphi+\dot{\psi} \cos \theta \cos \varphi ; \\
& \omega_{z}=\dot{\theta} \cos \varphi-\dot{\psi} \cos \theta \sin \varphi
\end{aligned}
$$

Проекции вектора углового ускорения на оси подвижной системы координат вычисляем по формулам:

$$
\varepsilon_{x}=\frac{d \omega_{x}}{d t}=\ddot{\varphi}+\ddot{\psi} \sin \theta+\dot{\psi} \dot{\theta} \cos \theta ;
$$

$\varepsilon_{y}=\frac{d \omega_{y}}{d t}=\ddot{\theta} \sin \varphi+\ddot{\psi} \cos \theta \cos \varphi+\dot{\theta} \dot{\varphi} \cos \varphi-$;

- $\dot{\psi} \dot{\theta} \sin \theta \cos \varphi-\dot{\psi} \dot{\varphi} \cos \theta \sin \varphi$

$$
\begin{gathered}
\varepsilon_{z}=\frac{d \omega_{z}}{d t}=\ddot{\theta} \cos \varphi-\ddot{\psi} \cos \theta \sin \varphi-\dot{\theta} \dot{\varphi} \sin \varphi+ \\
+\dot{\psi} \dot{\theta} \sin \theta \sin \varphi-\dot{\psi} \dot{\varphi} \cos \theta \cos \varphi .
\end{gathered}
$$

Результаты моделирования на рис. 4 а, б процесса поворота колец Карданового подвеса на углы $\psi, \theta$ за время $t(s)$ показывают наличие колебаний вследствие малого момента сил трения качения в опорах колец. Этот эффект однозначно указывает на необходимость применения демпферов для гашения колебаний. Гидравлические демпферы, осуществляющие преобразование энергии колебаний в силы жидкостного трения, давно применяются в пневматических приводах промышленных роботов, выпускаются серийно и как комплектующие изделия могут быть применены для уменьшения амплитуды колебаний колец подвеса.

Как видно на рис. 4 в, угол $\gamma$ между вертикалью и радиус-вектором: центр подвеса $\rightarrow$ центр масс компрессора постепенно смещается, и сопла компрессора занимают вертикальное положение, т.е. по линии гравитационной нагрузки, что и требовалось доказать установкой генератора тяги на Кардановом подвесе.

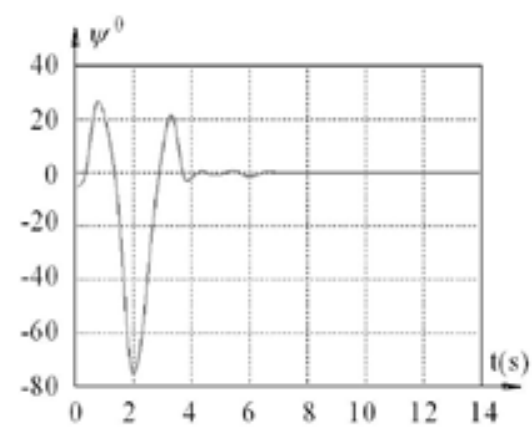

a

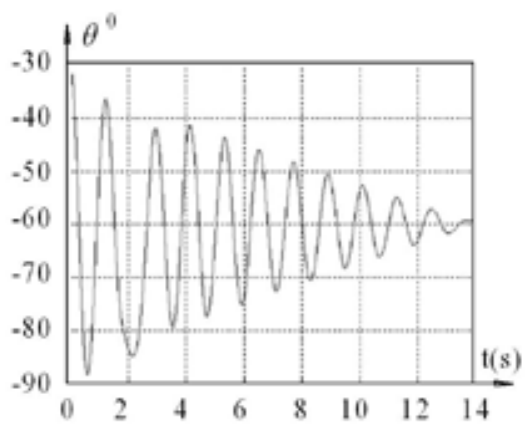

$\sigma$

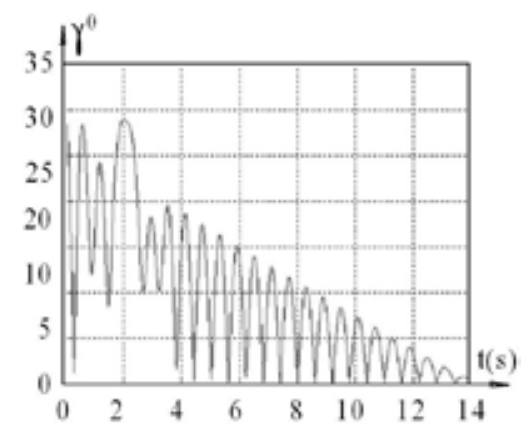

B

Рис. 4. Изменения углов поворота $\psi, \theta, \gamma$ колец Карданового подвеса 
Динамический анализ подвеса генератора тяги. Для исследования движения подвеса генератора тяги используем уравнение Лагранжа 2-го рода:

$$
\frac{d}{d t}\left(\frac{\partial T}{\partial \dot{q}_{i}}\right)-\frac{\partial T}{\partial q_{i}}=Q_{i}, \quad u=1,2, \ldots n,
$$

где $q_{i}$ - обобщённые координаты; $\dot{q}_{i}$ - обобщённые скорости; $Q_{i}$ - обобщённая сила, которая отвечает обобщённой координате $q_{i}$; $T=T\left(q_{i}, \dot{q}_{i}, t\right)$ - выражение кинетической энергии механической системы. Для данного случая при $q_{1}=\psi ; q_{2}=\theta ; q_{3}=\varphi$ система уравнений (5) будет иметь вид:

$$
\begin{aligned}
& \frac{d}{d t}\left(\frac{\partial T}{\partial \dot{\psi}}\right)-\frac{\partial T}{\partial \psi}=Q_{\psi} \\
& \frac{d}{d t}\left(\frac{\partial T}{\partial \dot{\theta}}\right)-\frac{\partial T}{\partial \theta}=Q_{\theta} . \\
& \frac{d}{d t}\left(\frac{\partial T}{\partial \dot{\varphi}}\right)-\frac{\partial T}{\partial \varphi}=Q_{\varphi}
\end{aligned}
$$

Механическая система подвеса состоит из трёх твёрдых тел, первое из них - это внутреннее кольцо 16 (рис. 2) диаметром $d_{b}$ и массой $m_{b}$. Это тело имеет две степени свободы и его положение полностью определяет углы $\psi$ и $\Theta$, а векторы угловой скорости указаны выше в выражениях (2). Поскольку оси системы координат $O x_{2} y_{2} z_{2}$ являются для кольца 16 главными осями инерции, то выражение кинетической энергии в (5) для данного кольца будет иметь вид:

$$
T_{1}=\frac{1}{2}\left(\left(\omega_{x 2}\right)^{2} I_{x 2}+\left(\omega_{y 2}\right)^{2} I_{y 2}+\left(\omega_{z 2}\right)^{2} I_{z 2}\right) .
$$

Определив осевые моменты инерции кольца $I_{\mathrm{x} 2}, I_{\mathrm{y} 2} I_{\mathrm{z} 2}$ (выражения которых для краткости здесь не приводим) при условии $y_{2}=0$, поскольку кольцо вращается вокруг этой оси, получим формулу кинетической энергии:

$$
T_{1}=\frac{m_{b} d_{b}^{2}}{16}\left((\dot{\psi})^{2}\left(1+\cos ^{2} \theta\right)+(\dot{\theta})^{2}\right) .
$$

Второе тело, т.е. генератор тяги (компрессор) имеет три степени свободы и его положение в пространстве целиком определяют углы $\psi, \theta, \varphi$, а проекции вектора угловой скорости описывают формулы (3). Тогда для компрессора выражение кинетической энергии можно записать как для тела, которое осуществляет сферическое движение:

$$
\begin{gathered}
T_{2}=\frac{1}{2}\left(\omega_{x}^{2} I_{x}+\omega_{y}^{2} I_{y}+\omega_{z}^{2} I_{z}-2 \omega_{x} \omega_{y} I_{x y}-\right. \\
\left.-2 \omega_{x} \omega_{z} I_{x z}-2 \omega_{y} \omega_{z} I_{y z}\right),
\end{gathered}
$$

где $I_{x}, I_{y}, I_{z}$ - осевые моменты инерции компрессора относительно осей системы отсчёта соединённой с компрессором; $I_{x y}, I_{x z}, I_{y z}$-центробежные моменты инерции компрессора.

И наконец, третье тело - внешнее кольцо 15 (рис. 2), диаметр которого $d_{3}$ и масса $m_{3}$. Это кольцо имеет одну степень свободы, оно осуществляет вращательное движение вокруг недвижимой оси кольца 14 и его положение полностью определяет угол $\psi$. Кинетическая энергия этого кольца равняется

$$
T_{3}=\frac{1}{2}(\dot{\psi})^{2} I_{3}=\frac{m_{3} d_{3}^{2}}{16}(\dot{\psi})^{2} .
$$

Таким образом, полная кинетическая энергия механической системы - Карданового подвеса с генератором тяги, равняется сумме найденных кинетических энергий отдельных тел:

$$
\begin{aligned}
& T=\frac{m_{b} d_{b}^{2}}{16}\left((\dot{\psi})^{2}\left(1+\cos ^{2} \theta\right)+(\dot{\theta})^{2}\right)+\frac{m_{3} d_{3}^{2}}{16}(\dot{\psi})^{2}+ \\
& +\frac{1}{2}\left(\omega_{x}^{2} I_{x}+\omega_{y}^{2} I_{y}+\omega_{z}^{2} I_{z}-2 \omega_{x} \omega_{y} I_{x y}-2 \omega_{x} \omega_{z} I_{x z}-2 \omega_{y} \omega_{z} I_{y z}\right)
\end{aligned}
$$

Определив частные производные в данном выражении, запишем проекции силы тяжести $\vec{G}_{2}$ генератора тяги на оси неподвижной системы координат $O x_{o} y_{o} z_{o}$ с учётом угла $\alpha$ - наклона мобильного робота к горизонту:

$$
\vec{G}_{2}=m_{2} g\left(\vec{i}_{o} \sin \alpha-\vec{j}_{o} \cos \alpha+0 \vec{k}_{o}\right),
$$

а также проекции силы тяжести $\vec{G}_{2}$ на оси системы координат $O x y z$, постоянно соединённой с компрессором:

$$
\begin{aligned}
& G_{2 x}=m_{2} g\left(a_{11} \sin \alpha-a_{21} \cos \alpha\right) \\
& G_{2 y}=m_{2} g\left(a_{12} \sin \alpha-a_{22} \cos \alpha\right) \\
& G_{2 z}=m_{2} g\left(a_{13} \sin \alpha-a_{23} \cos \alpha\right)
\end{aligned}
$$

Аналогично определяются проекции сил веса внешнего 15 и внутреннего 16 колец Карданового подвеса. Далее, предварительно определив момент силы $\vec{G}_{2}$ относительно центра «O» подвеса и проекции этого момента на оси системы координат $O x y z$, соединённой с компрессором, для определения обобщённых сил $Q_{q i}$, которые входят в правые части уравнений Лагранжа 2-го рода (6), будем предоставлять поочерёдно каждой обобщённой координате возможное перемещение $\delta_{q i}$ (т.е. малое перемещение, которое разрешается наложенными на механическую систему связями) и вычислять при этом возможную работу $\delta A_{q i}$. В итоге, после ряда преобразований, сведём задачу динамики Карданового подвеса с генератором тяги к решению системы линейных дифференциальных уравнений: 


$$
\left\{\begin{array} { l } 
{ \frac { d v _ { 1 } } { d t } = c _ { 1 1 } D _ { 1 } + c _ { 1 2 } D _ { 2 } + c _ { 1 3 } D _ { 3 } ; } \\
{ \frac { d v _ { 2 } } { d t } = c _ { 2 1 } D _ { 1 } + c _ { 2 2 } D _ { 2 } + c _ { 2 3 } D _ { 3 } ; } \\
{ \frac { d v _ { 3 } } { d t } = c _ { 3 1 } D _ { 1 } + c _ { 3 2 } D _ { 2 } + c _ { 3 3 } D _ { 3 } ; }
\end{array} \quad \left\{\begin{array}{l}
\frac{d \psi}{d t}=v_{1} \\
\frac{d \theta}{d t}=v_{2} \\
\frac{d \varphi}{d t}=v_{3}
\end{array}\right.\right.
$$

где обозначено $D_{1}=Q_{\psi}-F_{1}+\frac{\partial T}{\partial \psi} ; D_{2}=Q_{\theta}-F_{2}+\frac{\partial T}{\partial \theta}$; $D_{3}=Q_{\varphi}-F_{3}+\frac{\partial T}{\partial \varphi} ; \dot{\psi}=v_{1} ; \quad \dot{\theta}=v_{2} ; \quad \dot{\varphi}=v_{3} ; F_{1,2,3}-$ выражения частных производных по углам поворота колец подвеса с учётом моментов сил $Q_{\psi}, Q_{\theta}, Q_{\varphi}$. Эта система дифференциальных уравнений решена числовым методом, в частности, методом Рунге-Кутта четвёртого порядка точности при заданных начальных значениях углов Эйлера-Крылова $\psi, \theta, \varphi$ (см. выше, рис. 2 и рис. 4$)$ и их производных по времени.

Расчёт силы генератора тяги. Объём методики расчёта этого определяющего параметра мобильного робота с генератором тяги превышает рамки данной публикации. Поэтому здесь ограничимся основными положениями её корректности и рабочими формулами для расчёта аэродинамической подъёмной силы, т.е. пневматической реактивной силы тяги, осуществляющей противодействие силе тяжести робота в контексте, указанном выше (см. 1-й абзац подраздела «Конструкция мобильного робота).

Как известно, процесс адиабатного истечения воздуха можно описать системой уравнений в дифференциальной форме. Уравнение изменения количества движения (импульса) за элементарный промежуток времени при условии пренебрежения силами трения, а для газа это допустимо, имеет вид:

$$
\rho u d u=-d P,
$$

где $\rho$ - плотность газа, кг $/ \mathrm{M}^{3} ; u$ - переменная скорость движения газа вдоль отверстия (сопла, см. рис. 2, поз. 19), м/с; $P$ - давление газа, Н/м². Уравнение баланса энергии с учётом того, что при адиабатном процессе отсутствует обмен тепловым потоком с внешней средой $(d q=0)[16]$ :

$$
C_{P} d T+\mu u d u=0,
$$

где $C_{p}$ - теплоёмкость одного моля газа при постоянном давлении, Дж/моль; $T$ - абсолютная температура, ${ }^{0}$ Кельвина; $\mu$ - масса одного моля газа, кг/моль.

Уравнение Клапейрона-Менделеева, применяемое с определённой точностью и к реальным газам, имеет вид:

$$
d\left(\frac{P}{\rho}\right)=\frac{R}{\mu} d T,
$$

где $R=8,32$ Дж/(град.моль) - универсальная газовая постоянная. Причём из физики известно, что $R=C_{P}-C_{V}, \quad\left(C_{V}-\quad\right.$ теплоёмкость одного моля газа при постоянном объёме, Дж/моль). Решив равенство (17) относительно $d T: d T=d\left(\frac{P}{\rho}\right) \frac{\mu}{R}=d\left(\frac{P}{\rho}\right) \frac{\mu}{C_{P}-C_{V}}$ и подставив в уравнение (16), получим выражение:

$$
\frac{k}{k-1} d\left(\frac{P}{\rho}\right)+u d u=0,
$$

где отношение теплоёмкостей $k=C_{P} / C_{V}$ (для воздуха $k=1,4)$. Интегрируем это дифференциальное уравнение:

$$
\begin{aligned}
& \int_{u_{1}}^{u_{2}} u d u=-\frac{k}{k-1} \int_{P_{1} / \rho_{1}}^{P_{2} / \rho_{2}} d\left(\frac{P}{\rho}\right) \text { или } \\
& \frac{u_{2}^{2}}{2}-\frac{u_{1}^{2}}{2}=-\frac{k}{k-1}\left(\frac{P_{2}}{\rho_{2}}-\frac{P_{1}}{\rho_{1}}\right),
\end{aligned}
$$

где $u_{1}, P_{1}, \rho_{1}$ - скорость, давление и плотность газа на входе в отверстие сопла соответственно; $u_{2}, P_{2}, \rho_{2}-$ скорость, давление и плотность газа на выходе из сопла. Из равенства (19) находим скорость $u_{2}$ (скорость $u_{1}$ очень мала и в дальнейшем ею пренебрегаем):

$$
u_{2}=\sqrt{\frac{2 k}{k-1} \frac{P_{1}}{\rho_{1}}\left(1-\frac{P_{2}}{P_{1}} \frac{\rho_{1}}{\rho_{2}}\right)} .
$$

Исключив из уравнений (15) и (18) элемент $u d u$, после преобразования получим дифференциальное уравнение, интегрирование и последующее потенцирование которого даст окончательно выражение:

$$
\left(\frac{\rho}{\rho_{1}}\right)^{k}=\frac{P}{P_{1}} \Rightarrow \frac{\rho_{2}}{\rho_{1}}=\left(\frac{P_{2}}{P_{1}}\right)^{1 / k} .
$$

Подставив выражение (21) в формулу (20), получим значение скорости газа $u_{2}$ на выходе из сопла согласно формуле Сен-Венана [17, с. 188-192]:

$$
u_{2}=\sqrt{\frac{2 k}{k-1} \frac{P_{1}}{\rho_{1}}\left(1-\left(\frac{P_{2}}{P_{1}}\right)^{\frac{k-1}{k}}\right)} .
$$

Массу газа, который вытекает через сопло за единицу времени, можно найти по известной формуле:

$$
M=\rho_{2} u_{2} F_{2},
$$

где $F_{2}$ - площадь поперечного сечения сопла на выходе газа, $M^{2}$. Зная массу истекающего 
газа $M$, можно вычислить подъёмную силу генератора тяги $G_{a}$ :

$$
G_{a}=M u_{2}+\left(P_{1}-P_{2}\right) F_{2} .
$$

Учитывая приведённые выше соотношения, формула (24) будет иметь окончательный вид:

$$
\begin{gathered}
G_{a}=\frac{2 k}{k-1} \frac{\rho_{a}}{\rho_{1}} P_{1} F_{2}\left(1-b^{\frac{k-1}{k}}\right)+\left(P_{1}-P_{a}\right) F_{2} \\
\text { при } \quad b=\frac{P_{a}}{P_{1}} \leq b_{\kappa p}
\end{gathered}
$$

$G_{a}=\frac{2}{k+1}\left(b_{\kappa p}\right)^{\frac{1}{k}} P_{1} F_{2}+\left(P_{1}-b_{\kappa p} P_{1}\right) F_{2}$ при $\mathrm{b}=\mathrm{b \kappa p}$,

где $P_{\text {a }}$ - атмосферное давление; $b_{\kappa p}-$ критическое отношение давлений газа. Известно, что скорость звука в газе исчисляется по формуле $a=\sqrt{k P / \rho}$, тогда можно записать следующее выражение:

$$
\frac{d F}{F}=\frac{d u}{u}\left(\frac{u^{2}}{a^{2}}-1\right) .
$$

Из равенства (26) видно, что, пока скорость истечения газа $u<a$, для роста скорости $(d u>0)$ необходимо, чтобы сопло суживалось $(d f<0)$. Скорость u будет возрастать до тех пор, пока не достигнет критического значения $u_{\kappa p}$, которое равняется скорости звука при данных параметрах газа, то есть

$$
u_{k p}=\sqrt{k \frac{P_{2}}{\rho_{2}}}=\sqrt{k \frac{P_{1}}{\rho_{1}} b^{\frac{k-1}{k}}} ; \quad b=\frac{P_{2}}{P_{1}},
$$

На рис. 5 показаны результаты моделирования полученных зависимостей в среде MATLAB для сопла с отверстием диаметром $d_{o}=0,003 \mathrm{M}$. Разумеется, эти данные требуют экспериментальной апробации, что является следующим этапом исследований. Однако даже в теоретической реализации полученные зависимости приемлемы для этапа технического проекта опытного образца мобильного робота с генератором реактивной тяги. График рис. 5 а иллюстрирует зависимость скорости $U(\mathrm{~m} / \mathrm{s})$ истечения газа от давления $P$. График рис. 5 б иллюстрирует вполне рентабельный расход сжатого воздуха для создания аэродинамической подъёмной силы, а на графиках рис. 5 в, где кривая $G_{1}$ иллюстрирует изменение силы тяги от скорости истечения сжатого воздуха, кривая $G_{2}$ - от разницы давлений, $G_{a}$ - отображает суммарную силу тяги, можно установить необходимое число сопел, а именно:

если задать $n$ отверстий диаметром $d$, то расход $M_{c}$ сжатого воздуха и величину силы тяги $G$ можно вычислить по формулам:

$$
M_{c}=M n\left(\frac{d}{d_{o}}\right)^{2} ; \quad G=G_{a} n\left(\frac{d}{d_{o}}\right)^{2},
$$

где значение $M$ и $G_{a}$ выбираем из приведённых графиков в зависимости от давления в камере компрессора.

Так, например, пусть число сопел равно $n=50 \mathrm{c}$ отверстиями диаметром $d=0,004$ (м) и давлением в камере $P_{1}=6 \cdot 10^{5}$ (Па). Тогда из графика находим $G_{a}=5,2(N)$ и суммарная сила реактивной тяги $G$ составит: $\quad G=5,2 \cdot 50 \cdot(0,004 / 0,003)^{2}=462(N)$. Этот частный пример свидетельствует о возможности промышленной эксплуатации мобильного робота массой не более 50 кг при условии регулирования силы реактивной тяги, как это описано выше. Следует учитывать, что увеличение числа сопел до сотен при одновременном уменьшении их диаметра, вплоть до десятых долей миллиметра, а это возможно применением лазерной технологии, способствует стабилизации положения мобильного робота произвольной ориентации вследствие обеспечения ламинарного потока в соплах и снижения турбулентности потока воздуха на выходе сопел генератора пневматической реактивной тяги.

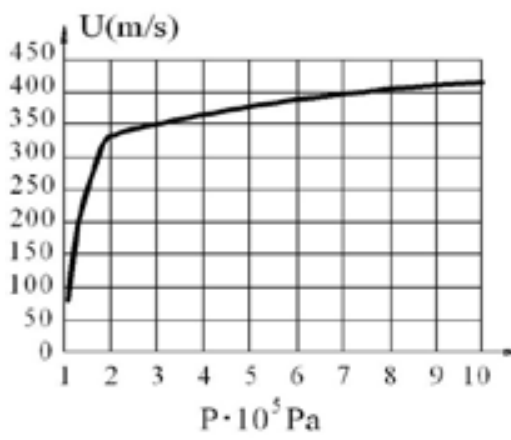

a

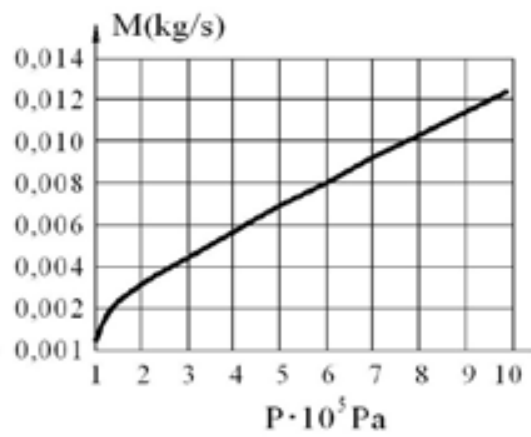

6

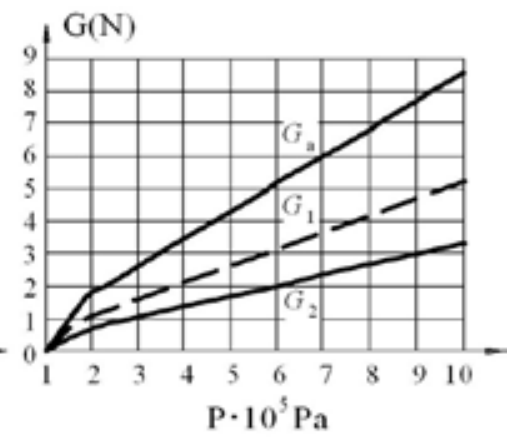

B

Рис. 5. Зависимости скорости истечения газа (a), расхода сжатого воздуха (б) и величины силы реактивной тяги (в) от давления истекающего воздуха 
Выводы. Применение пневматического генератора тяги как средства противодействия гравитационной силе позволяет при регулировании силы тяги в зависимости от угла наклона робота к горизонту повысить надёжность удержания робота на поверхности произвольной ориентации, что, в свою очередь, предоставляет возможность уменьшения мощности приводов для сцепления робота с поверхностью перемещения.

Полученные аналитические зависимости предшествуют их экспериментальной апробации, однако на данном этапе исследований могут быть использованы для технического проекта опытного образца мобильного робота с генератором пневматической реактивной тяги.

Предлагаемый подход синтеза мобильного робота произвольной ориентации позволяет за счёт уменьшения суммарной мощности приводов сцепления пропорционально уменьшить вес робота, а освободившейся энергетический ресурс направить на повышение эффективности выполнения как транспортных, так и технологических операций, выполняемых роботом в различных областях промышленности.

\section{Список литературы:}

1. Raju D. Dethe, Jaju S.B. Developments in wall climbing robots: a review. International journal of engineering research and general science. Volume 2, issue 3. 2014. p. 37, fig. 6.

2. Градецкий В.Г., Вешников В.Б., Калиниченко С.В., Кравчук Л.Н. Управляемое движение мобильных роботов по произвольно ориентированным в пространстве поверхностям. Москва : Наука, 2001. 360 с.

3. Черноусько Ф.Л., Болотник Н.Н., Градецкий В.Г. Мобильные роботы: исследования, разработки, перспективы. 2018. URL: http://www.ras.ru/news/shownews.aspx?id=f5c75bcf-2fa5-40e6-b0674492f0c5ab22\&print=1 (дата обращения: 09.02.2019).

4. Чащухин В.Г. Исследование параметров движения робота со скользящим уплотнением. Вестник Нижегородского университета им. Н.И. Лобачевского, 2011, № 4 (2). С. 347-349.

5. Climber robot: Patent 5551525 US: Int. Cl. B62D 57/032. Appl. No 293498; filed. 19.08.1994; publ. 03.09.1996. $17 \mathrm{p}$.

6. Поліщук М.М. Модернізація вакуумного захвату мобільного крокуючого робота. Mechanics and Advanced Technologies. № 2 (80). 2017. C. 59-64.

7. Saundersa A., Goldmanb D.I., Fullb R.J. and Buehlera M. The RiSE Climbing Robot: Body and Leg Design. Boston Dynamics, Unmanned Systems Technology VIII. Proc. of SPIE Vol. 6230. 2005. P. 11-13.

8. Tin Lun Lam, Yangsheng Xu. Tree Climbing Robot: Design, Kinematics and Motion Planning. Springer Heidelberg New York. 2012. P. 37-46.

9. Кузнєцов Ю.М., Шибецький В.Ю. Робототехнічні системи і комплекси фармацевтичного та біотехнологічного виробництв: навчальний посібник / під заг. ред. Ю.М. Кузнєцова. Київ : ГНОЗІС, 2012. 335 с.

10. Polishchuk M., Oliinyk V. Mobile climbing robot with elastic energy accumulators. Mechanics and Advanced Technologies. 2018. № 1. C. 116-122.

11. Climbing vehicle with suspension mechanism: pat. 9428231 US: Int.Cl. B62D 55/00. Appl. № 179464; filed. 12.02.2014; publ. 30.08.2016. 26 p.

12. Патент РФ на полезную модель 101683 МКИ В62D 57/04. Транспортное устройство для движения по вертикальным металлическим поверхностям; заяв. 29.09.2010; опубл. 27.01.2011. Бюл. № 3.

13. Yehya M., Hussain S., Wasim A., Jahanzaib M. and Abdalla H. Cost Effective and Light Weight Unipolar Electroadhesion Pad Technology for Adhesion Mechanism of Wall Climbing Robot. International Journal of Robotics and Mechatronics. Vol. 2, No. 1. 2014. P. 1-10.

14. Заявка № а201805661 на видачу патенту UA МПК B62D 57/024. Антигравітаційний мобільний робот Поліщука; заявл. 22.05.2018. Київ : Укрпатент, 2018. 16 с.

15. Polishchuk M., Opashnianskyi M., Suyazov N. Walking Mobile Robot of Arbitrary Orientation. International Journal of Engineering and Manufacturing (IJEM). Vol. 8, No. 3. 2018. P. 1-11.

16. Истечение газов и паров через сопловые каналы. 2018. URL: http://ispu.ru/files/u2/book2/TD2_1906/4.html (дата обращения: 31.07.2018).

17. Повх И.Л. Техническая гидромеханика. 2-е изд. Ленинград : «Машиностроение», 1976. 505 с.

\section{ТЕОРЕТИЧНЕ ОБГРУНТУВАННЯ ФУНКЦОНУВАННЯ АНТИГРАВІТАЦІЙНОГО МОБІЛЬНОГО РОБОТА}

У роботі викладено опис принщипово нової конструкції мобільного робота з генератором пневматичної реактивної тяги, установленому на Кардановому підвісі. Наведено синтез конструкцій виконавчих механізмів робота й математичні моделі їх функціонування. Технічний розв'язок спрямований на компенсацію або повне подолання гравітаційного навантаження мобільного робота довільної орі- 
єнтації під час виконання транспортних і технологічних операцій. У сукупності запропоновані технічні рішення сприяють підвищенню надійності втримання мобільних роботів на поверхні довільної орієнтації в просторі за їх експлуатачії в різних галузях промисловості.

Ключові слова: мобільні роботи, крокуючі механізми, роботи вертикального переміщення, альпіністські роботи.

\section{THEORETICAL SUBSTANTIATION OF FUNCTIONING}

ANTIGRAVITATIONAL MOBILE ROBOT

The paper describes a fundamentally new design of a mobile robot with a generator of pneumatic jet traction mounted on a Cardan suspension. Synthesis of the robot execution mechanisms and mathematical models of their functioning are given. The technical solution is aimed at compensating or completely overcoming the gravitational load of a mobile robot of arbitrary orientation when performing transport and technological operations. The proposed solutions are aimed at increasing the reliability of retention of mobile robots on the surface of arbitrary orientation in space.

Key words: mobile robots, walking machines, robots of vertical movement, climbing robots. 